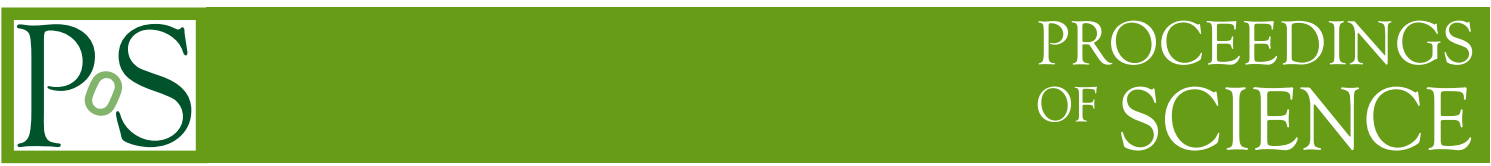

\title{
Long term monitoring of INTEGRAL sources with Swift/BAT
}

\author{
Gerald Skinner, ${ }^{a b c}$ Jack Tueller, ${ }^{c}$ Volker Beckmann, ${ }^{d}$ Robin Corbet, ${ }^{e b c}$, Sean Farrell, ${ }^{f}$ \\ Hans Krimm, ${ }^{g b c}$ and Craig Markwardt ${ }^{a b c}$ \\ ${ }^{a}$ University of Maryland, College Park, MD 20742, USA \\ ${ }^{b}$ CRESST, Greenbelt, MD 20771, USA \\ ${ }^{c}$ NASA-GSFC, Code 661, Greenbelt, MD 20771, USA \\ ${ }^{d}$ ISDC Data Centre for Astrophysics, Versoix, Switzerland \\ ${ }^{e}$ University of Maryland, BC, 1000 Hilltop Circle Baltimore, MD 21250, USA \\ ${ }^{f}$ CESR, 9, av. du Colonel Roche, Cedex 4, Toulouse 31028, France \\ ${ }^{g}$ USRA, Columbia, MD 21044, USA \\ E-mail: skinner@milkyway.gsfc.nasa.gov, Jack.Tueller@nasa.gov, \\ corbet@milkyway.gsfc.nasa.gov, Volker.Beckmann@unige.ch. \\ Sean.Farrellecesr.fr, krimmemilkyway.gsfc.nasa.gov, \\ Craig.Markwardtenasa.gov
}

\begin{abstract}
INTEGRAL has discovered many previously unknown hard X-ray sources, particularly in the Galactic plane. Typically the INTEGRAL observations are deep but the sources are within the field of view only for a comparatively short time. A given sky direction is usually within the field of view of the BAT instrument on Swift $\sim 10 \%$ of the time and $\sim 85 \%$ of the sky is usually observed every day. Thus BAT is able to provide complementary hard-X-ray information on the long-term behavior of sources. We report new results on INTEGRAL and other sources, including orbital, super-orbital and other periodicities and spectral evolution histories.
\end{abstract}

7th INTEGRAL Workshop

September 8-11 2008

Copenhagen, Denmark 


\section{Introduction}

INTEGRAL is an observatory mission executing a planned program of observations of specific areas of sky. The Burst Alert Telescope (BAT) onboard the Swift satellite monitors $\sim 1 / 6$ of the entire sky in order to detect and locate Gamma-ray bursts (GRBs). During the time that it is awaiting gamma-ray bursts BAT collects a huge amount of "survey" data on the hard X-ray sky. Its very large field of view ( $\sim 2 \mathrm{sr}$; Fig. 1) and high sensitivity in the $14-150 \mathrm{keV}$ band make BAT a very powerful tool for following the long term hard X-ray behaviour of sources such as those being found by INTEGRAL

The Swift/BAT survey mode data provide information on the spectral and temporal evolution of sources that is complementary to that from INTEGRAL. If a source is within the field of view of BAT and of IBIS (or SPI) for the same exposure time, the sensitivity of BAT is inferior. However the wide field of view of BAT and the frequently changing and widely distributed pointing directions of the Swift spacecraft mean that a given part of the sky is within its field of view for much longer. The long-term sensitivities of the instruments are thus comparable, though the INTEGRAL observing program leads to better coverage of the Galactic bulge while BAT achieves higher sensitivity for most extragalactic fields. Table 1 compares some key parameters for the Swift BAT and INTEGRAL IBIS-ISGRI/SPI instruments.

The Swift/BAT Hard X-ray Transient Monitor [1] $]^{1}$ provides rapid access to intensities of sources on short timescales. The data used in the Transient Monitor analysis are however limited in two ways - only a single energy band (14-50 keV) is available and the form of the data do not allow all of the corrections possible in the full survey analysis, leading to systematic errors that can become important when data for many days is combined together.

Reduction of the full survey data for the first 36 months of the Swift mission (2004 Dec to 2007 Nov) has recently been completed. Although the analysis is largely directed towards getting the best possible sensitivity by combining all of the data available, a by-product of the work is 8 energy channel information on the intensities of over 500 sources that were included in a search catalogue (see Table 2). The time resolution is that of a single pointing which typically lasts about 10 minutes (median value $650 \mathrm{~s}$, Fig. 2). For a given source, data are typically obtained on 80$90 \%$ of days and in most cases there are $\sim 10^{7} \mathrm{~s}$ ( $\equiv 115$ days continuous) of observation with the source within the field of view. All of the data selection and corrections that are applied in the survey analysis have been applied in extracting the data. This treatment has been shown to reduce systematic errors to a low level even when very long integration times are used. We present here some sample results to indicate the potential of this database.

\section{Examples of results that can be extracted from the Swift/BAT snapshot database}

\subsection{Example 1. Long term light curve of an extragalactic source : IGR J21247+5058}

IGR J21247+5058 is identified with a $z=0.02$ galaxy at the core of an FRII radio source $4 \mathrm{C} 40.55$. Optical spectroscopy is confused by a chance alignment with a foreground star and the galaxy has been variously described as a Sy 2 or a BLRG. ([2], and references therein). The source

\footnotetext{
${ }^{1}$ http://swift.gsfc.nasa.gov/docs/swift/results/transients
} 


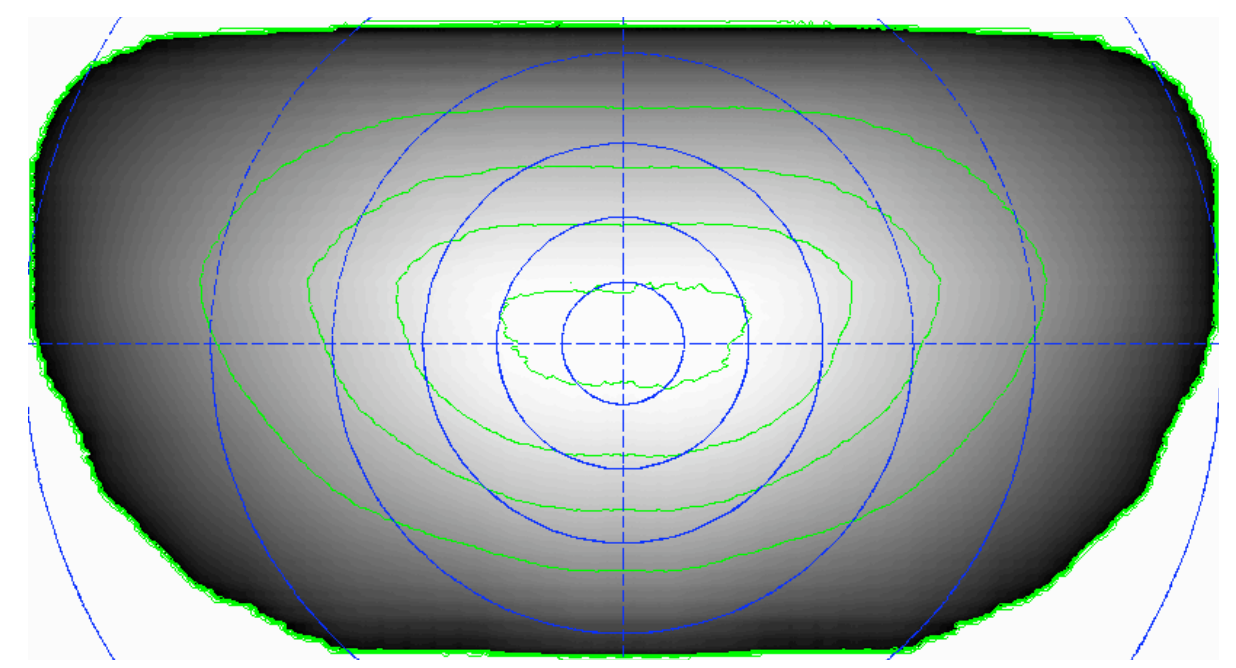

Figure 1: The instantaneous field of view of the Swift/BAT coded mask telescope. The circles indicate off-axis angles of $10^{\circ}, 20^{\circ} \ldots 60^{\circ}$. The contours correspond to $100,75,50,25,0 \%$ effective area.

\begin{tabular}{|c|c|c|c|c|}
\hline & Units & \multicolumn{2}{|c|}{ INTEGRAL } & Swift \\
\hline \multirow{2}{*}{$\begin{array}{l}\text { Orbital period } \\
\text { Time pointed at }|b|<14.5^{\circ} \\
\qquad(25 \% \text { of sky })\end{array}$} & \multirow[t]{2}{*}{ Days } & \multicolumn{2}{|c|}{$\begin{array}{c}3 \\
71 \%\end{array}$} & $\begin{array}{l}0.067 \\
29 \%\end{array}$ \\
\hline & & IBIS-ISGRI & SPI & BAT \\
\hline Instantaneous Field of view & $\begin{array}{c}\text { steradians } \\
\text { (to } 10 \% \text { coding) }\end{array}$ & 0.18 & 0.22 & 1.8 \\
\hline $\begin{array}{l}\text { Angular resolution } \\
\text { Energy range }\end{array}$ & $\begin{array}{c}\text { Arc minutes } \\
\mathrm{keV}\end{array}$ & $\begin{array}{c}12 \\
15-1000\end{array}$ & $\begin{array}{c}180 \\
20-8000\end{array}$ & $\begin{array}{c}22 \\
14-195\end{array}$ \\
\hline
\end{tabular}

Table 1: Comparison of Swift/BAT and the INTEGRAL gamma-ray instruments

\begin{tabular}{lc}
\hline Source positions examined & 533 \\
Measurements per source & $\sim 11000$ \\
Average measurement duration & $536 \mathrm{~s}$ \\
Observation time per source & $\sim 10^{7} \mathrm{~s}$ \\
Fraction of time for which data available on a given source & $10.5 \%$ \\
Number of 8 channel spectra & 6.4 million \\
Energy range bands (keV) & $14-20,20-24,24-35,35-50$, \\
& $50-75,75-100,100-150,150-195$ \\
\hline
\end{tabular}

Table 2: The Swift/BAT 36 month survey source database - some statistics and information 
Distribution of BAT survey time-bin durations for a typical source

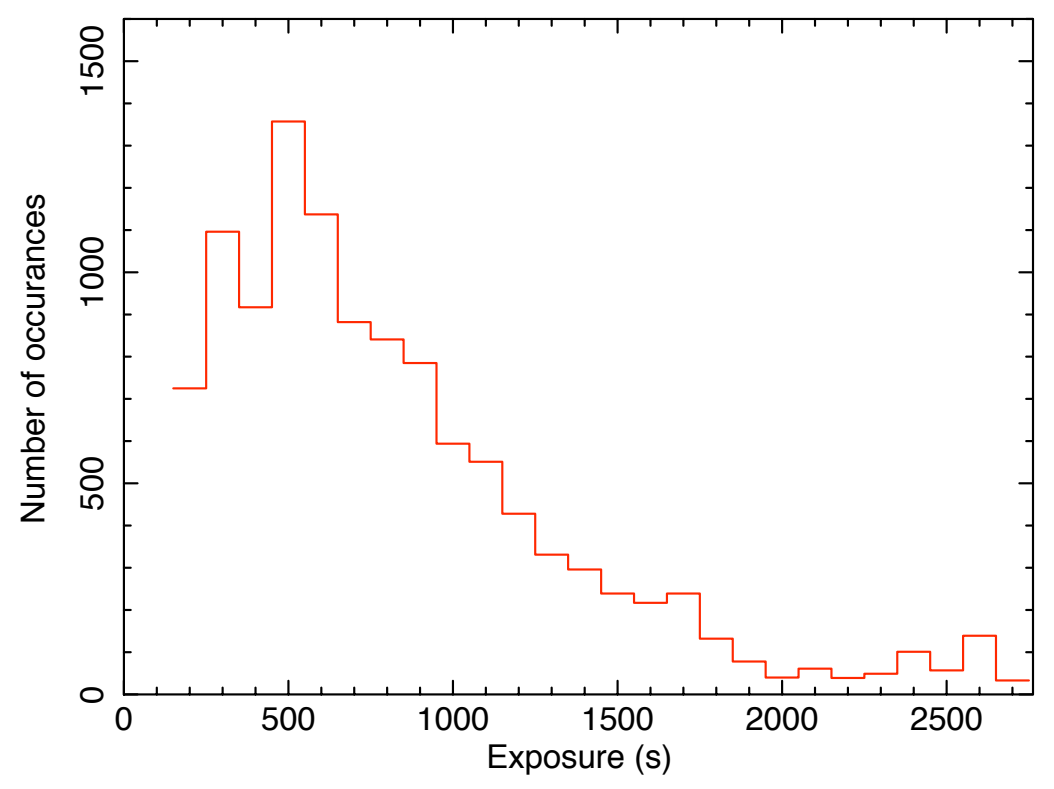

Figure 2: The distribution of BAT survey time-bin durations for a typical source

is very strongly detected in the Swift/BAT survey and a light curve shows both long term evolution and shorter timescale variability with a tendency for a rise over several months to be followed by a relatively sudden decrease (Fig. 3).

\subsection{Example 2. Spectral variability over $10^{7} \mathrm{~s}$}

With typically $\sim 100008$-band spectra available per source, relatively rare spectral states are sampled. Fig. 4 shows results from Sco X-1 which made only occasional excursions away from the state in which it was observed for most of the time. In most of these it was towards the highhard state. Observations in the high-soft state were comparatively rare. For more information and corresponding measurements with INTEGRAL, see [3].

\subsection{Example 3. Catching rare events such as occasional spikes in emission.}

IGR J18483-0311 was detected by INTEGRAL [4] and later 5 more outbursts were detected and it was noticed that its brief flares were separated by multiples of 18.52 days [5]. With much denser sampling than INTEGRAL, BAT reveals numerous further outbursts (Fig. 5). The periodicity is confirmed and the period can be refined - see Figs. 6,7. By combining these results with those from INTEGRAL, a provisional measurement of $(18.518 \pm 0.005)$ days is obtained. In this case the period appears to be strict and is presumably orbital in origin.

\subsection{Example 4. Orbital and Super-orbital lightcurves.}

A super-orbital period of 46 days has been described in $4 \mathrm{U} 1636-536$ [6]. Analysis of the 


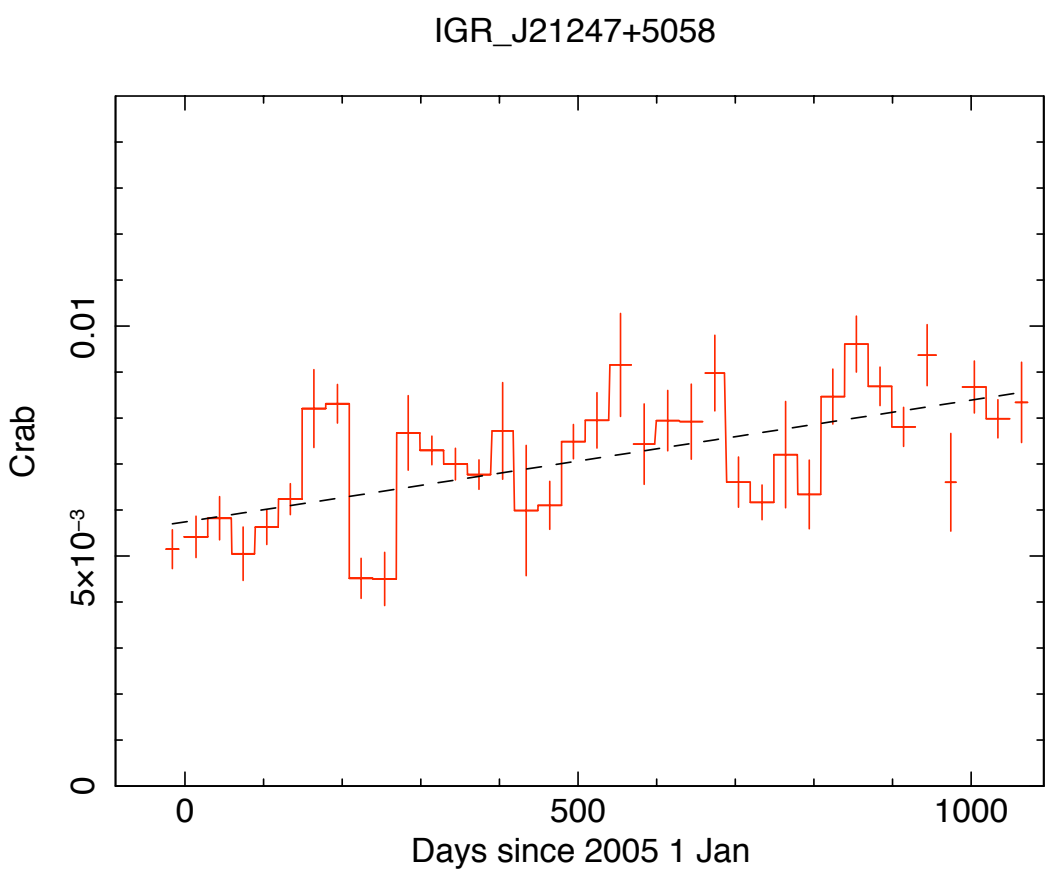

Figure 3: A BAT 14-195 keV light-curve of the extragalactic hard X-ray source associated with 4C40.55. The dashed line is a linear fit to the data.

Sco X-1

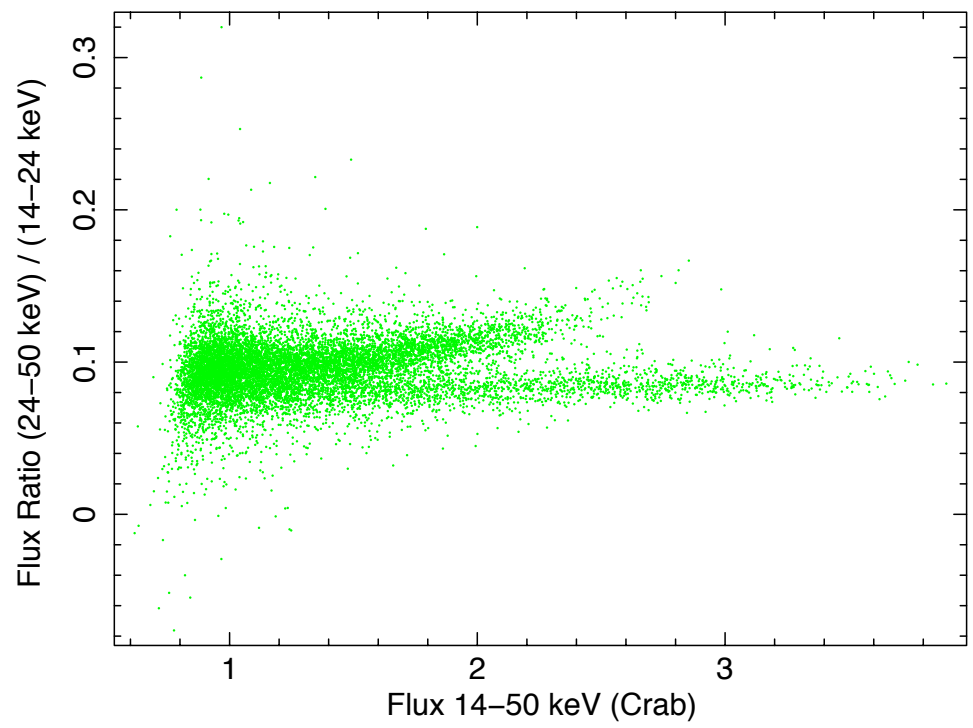

Figure 4: A colour-intensity plot for Sco-X-1 with more than $10^{4}$ samples. The lower branch, corresponding to a high-soft state was visited in comparatively few of the observations compared with the upper, high-hard, branch. 
IGR_J18483-0311

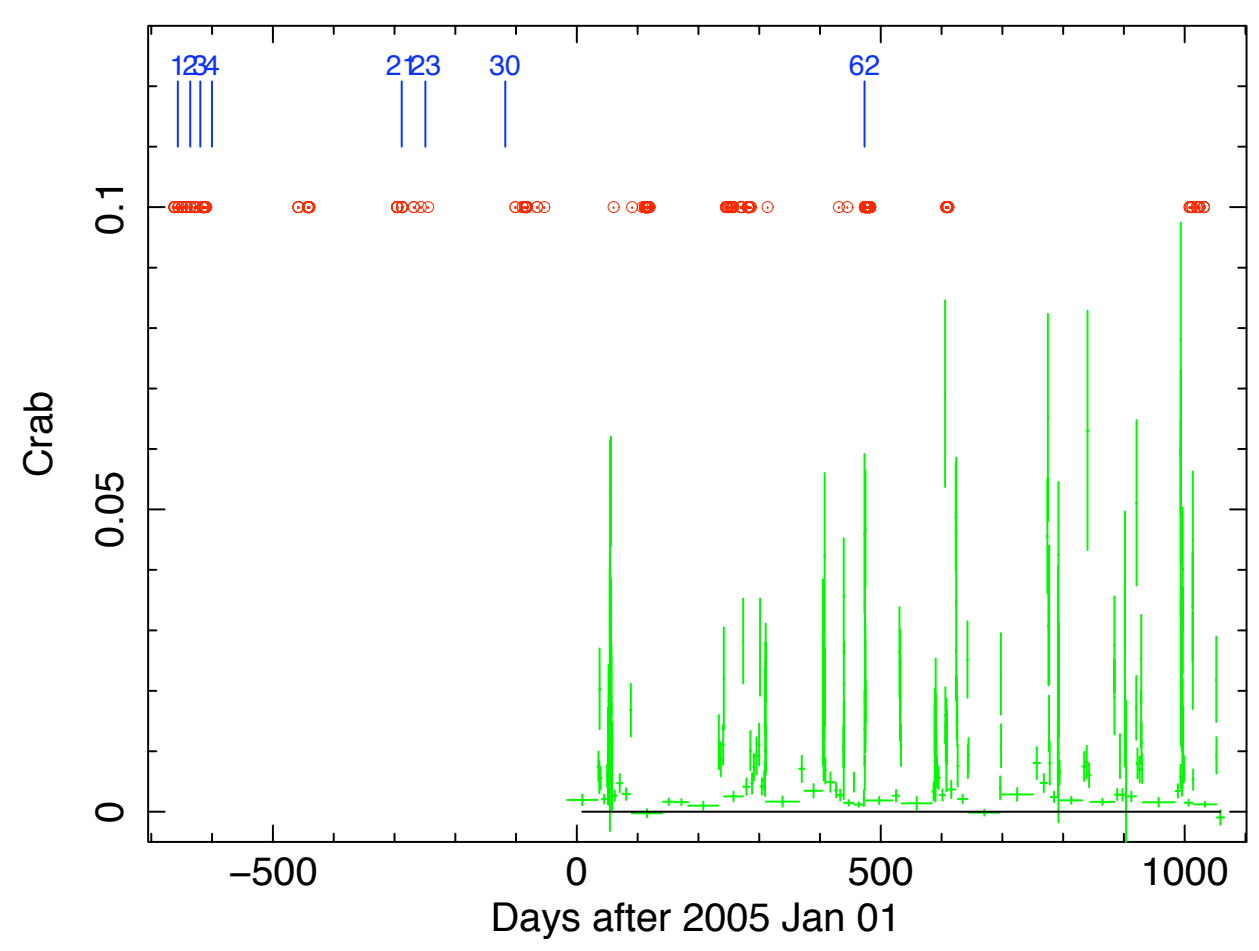

Figure 5: Hard X-ray (14-195 keV) observations of periodic flares from IGR J1843-0311. Swift coverage is much more complete than that of INTEGRAL (red circles) leading to the discovery of many more events than the 8 reported from IBIS observations [5] (marked by the cycle numbers).

hard X-ray light curve during the first 36 months of the Swift mission shows that a period of 42 days better describes the data (Fig. 8a). However a dynamic periodogram ( Fig. 9a) and comparison of the light curve with a 42 days sinusoid (Fig. 10) show that the outbursts are far from strictly periodic and can occur at any phase. It is clear that the period evolved during the observations. Remarkably, low energy X-ray data observed over the same range of times with the RXTE ASM show modulation inverted in phase compared with that in hard X-rays (Fig. 8b), emphasizing the importance of long term monitoring at higher energies as well as lower ones. The phase shift between soft and hard bands in 4U 1636-536 has been speculatively linked to transient jet formation [7].

A dynamic hard X-ray periodogram of SMC X-1 (Fig. 9b) clearly shows both the $3.89 \mathrm{~d}$ orbital period and the known super-orbital modulation [8,9]. Again the latter period clearly evolves during 3 years covered by the data.

Swift/BAT is the only current instrument that can provide the long-term, high-coverage, observations for making dynamic periodograms of this sort in the hard X-ray band.

\section{Conclusions}

The data from Swift/BAT provide a resource that is unique in the hard X-ray/soft gamma-ray 
IGR_J18483-0311

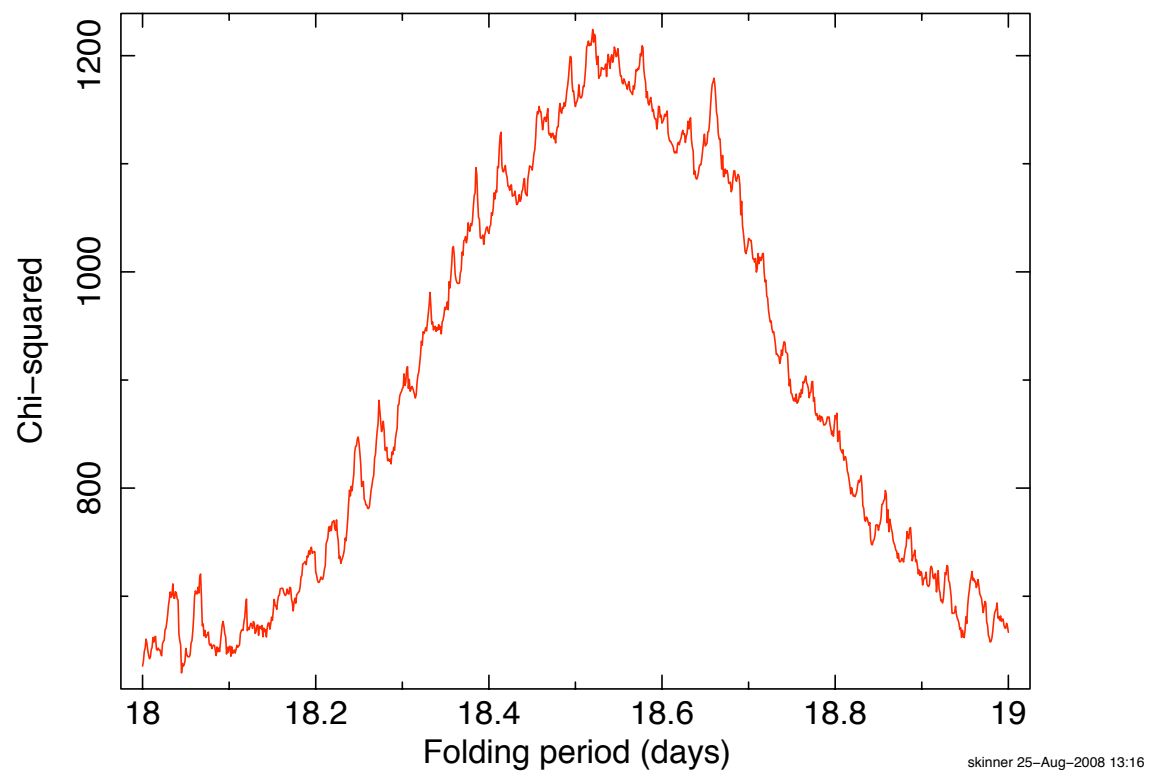

Figure 6: The $\chi^{2}$ as a function of trial period for the IGR J18483-0311 data.

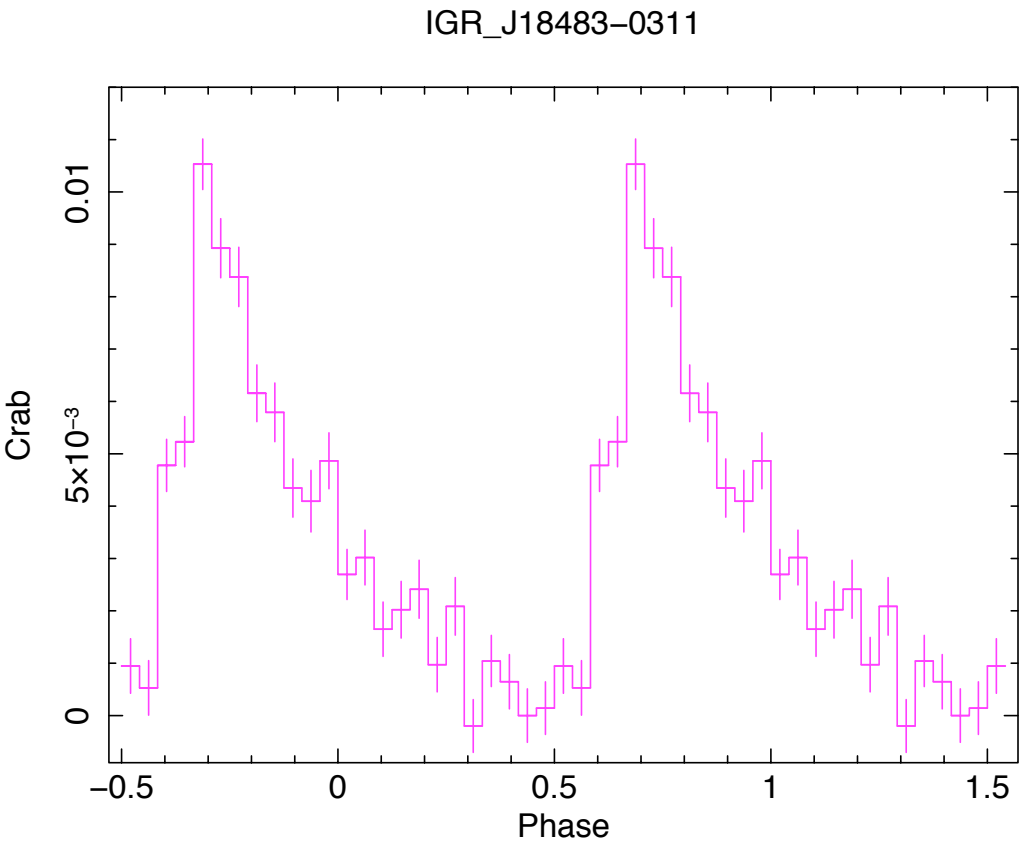

Figure 7: The folded BAT hard X-ray (14-195 keV) light curve for IGR J1843-0311 with a trial period of $18.517 \mathrm{~d}$. (Note error bars here are based on statistical errors and do not include the variance in the source intensity). 


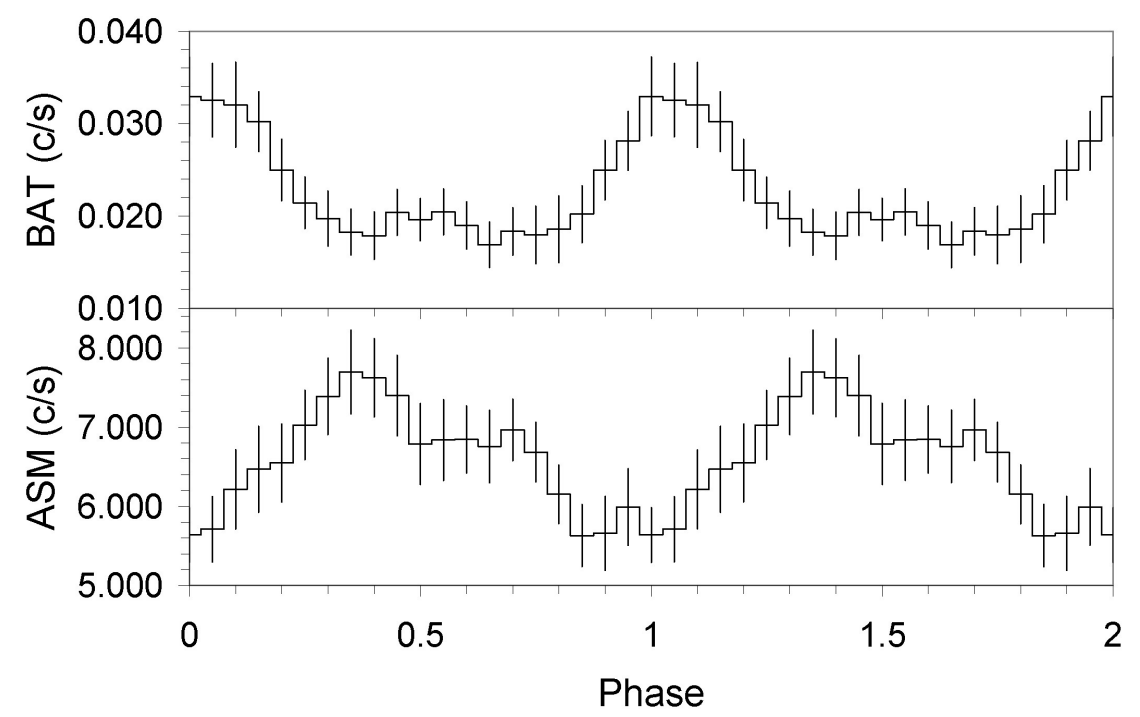

Figure 8: (a) The folded BAT hard X-ray (14-195 keV) light curve for 4U 1636-536. (b) The corresponding low energy X-ray curve from the RXTE ASM (1-12 keV). Again the error bars are statistical only.
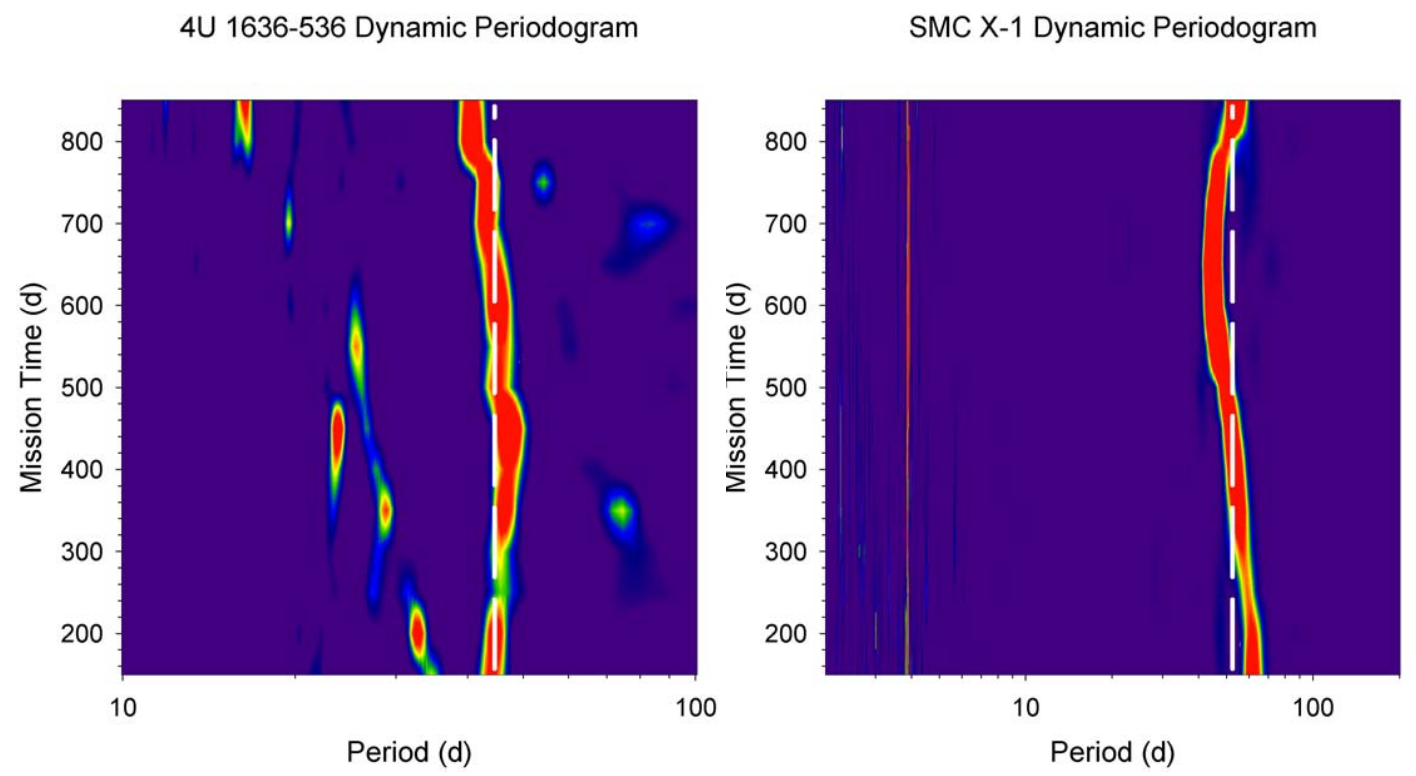

Figure 9: (a) A dynamic periodogram for $4 \mathrm{U}$ 1636-536 from BAT data. The dashed white line indicates the nominal period of 42 days. (b) The corresponding dynamic periodogram for SMC X-1. The 3.89 day orbital period is clearly visible. The dashed white line indicates a nominal super-orbital period of 52 days. 


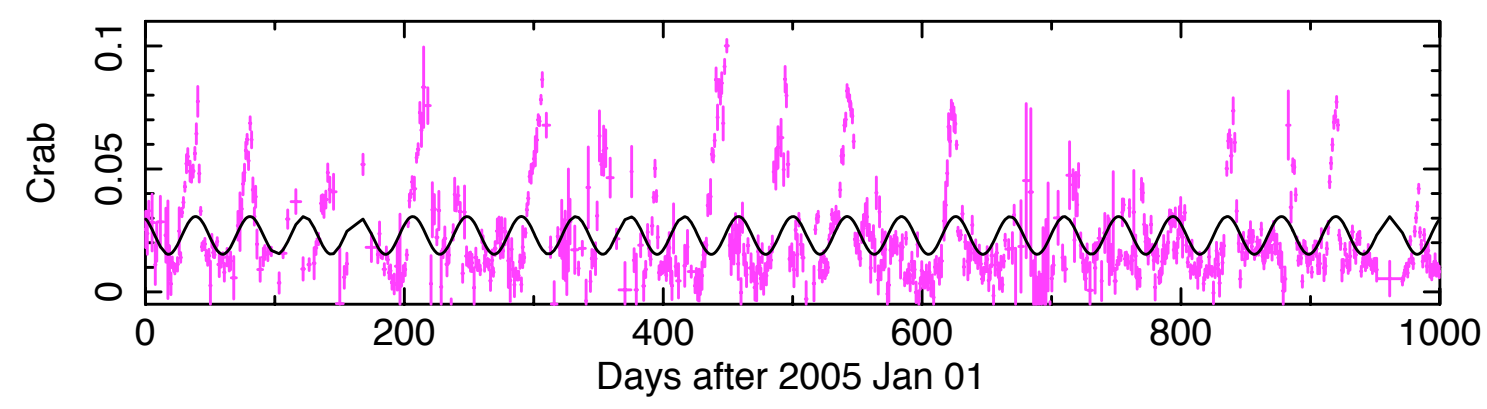

Figure 10: The BAT 14-195 keV light curve of 4U 1636-536 compared with a sinusoid with a constant period of 42.0 days, the best value found by folding the whole data set on various periods.

band and that is complementary to the INTEGRAL observations. Although the sensitivity for a given observing time with the source within the field of view is not as good as that of IBIS, the source coverage is vastly superior. It provides long-term monitoring of hard X-ray/soft gammaray sources, allowing the study of broad-band spectral variability over long time-scales, and of super-orbital variability in hard X-rays. It can provide a unique hard X-ray trigger for observations in other wavebands or for detailed follow-up X-/gamma-ray observations with the instruments of INTEGRAL.

\section{References}

[1] H. A. Krimm et al. Status of the Swift/BAT Hard X-ray Transient Monitor, AAS/High Energy Astrophysics Division 10 (2008) \#07.01

[2] M. Molina et al. Broad-band X-ray spectrum of the newly discovered broad-line radio galaxy IGR J21247+5058, MNRAS 382 (2007) 937

[3] S. J. Sturner \& C. R. Shrader, The Hard X-Ray Emission From Scorpius X-1 As Seen By INTEGRAL, These proceedings D07-37

[4] M. Chernyakova et al. IGR J18483-0311, The Astronomer's Telegram 157 (2003)

[5] V. Sguera et al. IGR J18483-0311: an accreting X-ray pulsar observed by INTEGRAL, A\&A 467 (2007) 249

[6] I. C. Shih et al. Periodic variability during the X-ray decline of 4U 1636-53, MNRAS 361 (2005) 602

[7] S. Farrell et al. Super-orbital variability in hard X-rays, MNRAS 393 (2009) 139

[8] P. Wojdowski et al. Quasi-periodic Occultation by a Precessing Accretion Disk and Other Variabilities of SMC X-1, ApJ 502 (1998) 253

[9] W. I. Clarkson et al. Long-term properties of accretion discs in X-ray binaries - I. The variable third period in SMC X-1, MNRAS 339 (2003) 447 